\title{
Potential of selected Senegalese Aedes spp. mosquitoes (Diptera: Culicidae) to transmit Zika virus
}

\author{
Cheikh Tidiane Diagne ${ }^{1,3^{*}}$, Diawo Diallo ${ }^{1}$ Oumar Faye², Yamar Ba ${ }^{1}$, Ousmane Faye ${ }^{2}$, Alioune Gaye ${ }^{1}$, Ibrahima Dia ${ }^{1}$,
} Ousmane Faye ${ }^{3}$, Scott C. Weaver ${ }^{4,5}$, Amadou Alpha Sall $^{2}$ and Mawlouth Diallo ${ }^{1}$

\begin{abstract}
Background: Zika virus (ZIKV; genus Flavivirus, family Flaviviridae) is an emerging virus of medical importance maintained in a zoonotic cycle between arboreal Aedes spp. mosquitoes and nonhuman primates in African and Asian forests. Serological evidence and virus isolations have demonstrated widespread distribution of the virus in Senegal. Several mosquito species have been found naturally infected by ZIKV but little is known about their vector competence.

Methods: We assessed the vector competence of Ae. aegypti from Kedougou and Dakar, Ae. unilineatus, Ae. vittatus and Ae. luteocephalus from Kedougou in Senegal for 6 ZIKV strains using experimental oral infection. Fully engorged female mosquitoes were maintained in an environmental chamber set at $27 \pm 1{ }^{\circ} \mathrm{C}$ and $80 \pm 5 \%$ Relative humidity. At day 5, 10 and 15 days post infection (dpi), individual mosquito saliva, legs/wings and bodies were tested for the presence of ZIKV genome using real time RT-PCR to estimate the infection, dissemination, and transmission rates.

Results: All the species tested were infected by all viral strains but only Ae. vittatus and Ae. luteocephalus were potentially capable of transmitting ZIKV after 15 dpi with 20 and $50 \%$ of mosquitoes, respectively, delivering epidemic (HD 78788) and prototype (MR 766) ZIKV strains in saliva.

Conclusion: All the species tested here were susceptible to oral infection of ZIKV but only a low proportion of Ae. vittatus and Ae. luteocephalus had the viral genome in their saliva and thus the potential to transmit the virus. Further investigations are needed on the vector competence of other species associated with ZIKV for better understanding of the ecology and epidemiology of this virus in Senegal.
\end{abstract}

Keywords: Aedes, Arboviruses, Oral infection, Senegal, Vector competence, West Africa, Zika virus

\section{Background}

Zika virus (ZIKV; genus Flavivirus, family Flaviviridae) is an emerging globally mosquito-borne pathogen of growing public health importance. The virus was first isolated in 1947 from a febrile sentinel rhesus monkey and one year later from Ae. africanus in Uganda [1]. Non-human primates (NHPs) were implicated as the vertebrates hosts of ZIKV in Africa and Asia [2]. The first well-documented report of human ZIKV infection

\footnotetext{
* Correspondence: ctdiagne@pasteur.sn

'Unité d'Entomologie Médicale, Institut Pasteur de Dakar, 36 Avenue Pasteur, BP 220, Dakar, Senegal

32épartement de Biologie Animale, Laboratoire d'Écologie Vectorielle et Parasitaire, Université Cheikh Anta Diop, Dakar, Senegal

Full list of author information is available at the end of the article
}

was in Uganda in 1964 when Simpson described his own occupationally acquired illness [3]. Subsequently, ZIKV has been recognized to be a cause of febrile illness in humans in Africa and Southeast Asia with symptoms including fever, headache, conjunctivitis, myalgia, rash, joint pains [4-6]. Serological evidence and virus isolations have demonstrated widespread distribution of the virus in Africa, the Indian subcontinent, Southeast Asia and most recently Micronesia and French Polynesia [3, 7-11]. The implication of Ae. aegypti in the urban transmission of ZIKV came first from field evidence including the high prevalence of anti-ZIKV antibodies in the urban population of Nigeria [12], the coincidance of peaks of human ZIKV infections and Ae. aegypti population in Indonesia 
[7] and the isolation of the virus from a pool of Ae. aegypti in Malaysia [13]. This implication of Ae. aegypti was confirmed by early experimental studies wich demonstrated the competence of this species to transmit ZIKV [14, 15]. The isolation of the virus from a pool of Ae. aegypti in Malaysia provided the first evidence of ZIKV transmission outside Africa.

ZIKV was also isolated from Ae. africanus and Ae. apicoargenteus in Uganda and the Central African Republic [16, 17]; from Ae. luteocephalus in Nigeria in 1969 and 1972 [12]; and from Ae. vittatus, Ae. furcifer, and Ae. aegypti in Cote d'Ivoire in 1999 [18].

In Senegal, the first evidence of ZIKV circulation was the isolation of a strain from Ae. luteocephalus collected in 1968 in the Saboya forest, $187 \mathrm{~km}$ from Dakar, in the western part of the country [15]. One year later, the virus was isolated from Ae. luteocephalus, Ae. furcifertaylori and An. gambiae s.l., and a human in Bandia located $65 \mathrm{~km}$ from Dakar. In Kedougou, Southeastern Senegal, 381 ZIKV isolates were collected as part of an entomological surveillance programme from 1972 to 2011, mainly from Ae. africanus, Ae. luteocephalus, Ae. furcifer, and Ae. taylori, 7 times from humans and twice from NHPs (Cercopithecus aethiops, Erythrocebus patas). Serological studies in 1988 and 1990 in the area showed that 10.1 and $2.8 \%$ of humans had Immunoglobuline M (IgM) antibodies to ZIKV [9].

Although several mosquito species have been found naturally infected by ZIKV, little is known about their vector competence. Hence, the purpose of the present study was to investigate vector competence of populations of Ae. aegypti from Dakar and Kedougou and Ae. unilineatus, Ae. vittatus, and Ae. luteocephalus from Kedougou for 6 ZIKV strains to take into account the high diversity of hosts (human, NHPs, and several mosquito species) and location from which ZIKV strains were isolated.

\section{Methods}

\section{Ethical approval}

The protocol of this study has been approved by the Senegalese National Ethic Committee under protocol SEN29/08; 2472/MSP/DS/DER. Because this study was done in collaboration with a team from the University of Texas Medical Branch (UTMB), the UTMB Institutional Animal Care and Use Committee also approved the animal experiments under protocol 02-09-068. UTMB complies with all applicable regulatory provisions of the U.S. Department of Agriculture (USDA)-Animal Welfare Act; the National Institutes of Health (NIH), Office of Laboratory Animal Welfare-Public Health Service (PHS) Policy on Human Care and Use of Laboratory Animals; the U.S. Government Principles for the Utilization and Care of Vertebrate Animals Used in Research,
Teaching, and Testing developed by the Interagency Research Animal Committee (IRAC), and other federal statutes and state regulations relating to animal research. The animal care and use program at UTMB conducts reviews involving animals in accordance with the Guide for the Care and Use of Laboratory Animals (2011) published by the National Research Council.

\section{Mosquitoes}

Table 1 describes the characteristics and geographic origin of the populations of Ae. aegypti, Ae. unilineatus, Ae. vittatus, and Ae. luteocephalus tested in this study. These species were chosen taken into account their abundance, anthrophophilic behaviour and association to ZIKV in the field [19]. For each population, several breeding habitats were prospected and collected eggs reared in the laboratory. Females F0 were fed several times on guinea pigs to obtain F1 generation eggs. These eggs were hatched and the larvae reared to F1 adults used in this study. This F1 generation were maintained exclusively with a $10 \%$ sucrose solution at $27{ }^{\circ} \mathrm{C}, 80 \%$ relative humidity (RH), 16:8 h (L:D) photoperiod.

\section{Virus strains}

Hosts origin, year of collection and passage histories of the six ZIKV strains used in this study are presented in Table 2. To prepare viral stocks, ZIKV isolates were amplified in Ae. albopictus C6/36 cells for a week at $27^{\circ} \mathrm{C}$ and infection progression was monitored using indirect immunofluorescence assay. Supernatant fluids were collected and viral titers estimated by serial 10 -fold dilutions on Vero cells [Plaque forming unit (PFU)/ml] as previously described by De Madrid and Porterfield [20]. Each virus stock was divided into $500 \mu \mathrm{l}$ aliquots and stored at $-80^{\circ} \mathrm{C}$ until use.

\section{Oral infection of mosquitoes}

Mosquito infection has been performed according to procedures already described [21]. Briefly, one-week-old females of each mosquito species that have never taken blood meal were starved for $24 \mathrm{~h}$ before the infectious meal. These females were allowed to feed through a chicken skin membrane by the artificial feeding method

Table 1 Mosquito species tested for vector competence for Zika virus

\begin{tabular}{llll}
\hline Species & Source $^{\mathrm{a}}$ & Habitat & Generation \\
\hline Ae. aegypti & Dakar & Domestic & $\mathrm{F}_{1}$ \\
& Kedougou & Sylvatic & $\mathrm{F}_{1}$ \\
Ae. unilineatus & Kedougou & Sylvatic & $\mathrm{F}_{1}$ \\
Ae. vittatus & Kedougou & Sylvatic & $\mathrm{F}_{1}$ \\
Ae. luteocephalus & Kedougou & Sylvatic & $\mathrm{F}_{1}$ \\
\hline
\end{tabular}

${ }^{\mathrm{a} A l l}$ mosquitoes were collected during 2012 
Table 2 Zika virus strains used for this study

\begin{tabular}{|c|c|c|c|c|}
\hline ZIKV strains & Host origin & Year of collection & Location & Passage history ${ }^{a}$ \\
\hline ArD 128000 & Mosquito (Ae. luteocephalus) & Oct. $11^{\text {th }} 1997$ & Kedougou (Senegal) & 6 \\
\hline ArD 132912 & Mosquito (Ae. dalzieli) & Nov. $20^{\text {th }} 1998$ & Kedougou (Senegal) & 4 \\
\hline ArD 157995 & Mosquito (Ae. dalzieli) & Nov. $17^{\text {th }} 2001$ & Kedougou (Senegal) & 6 \\
\hline ArD 165522 & Mosquito (Ae. vittatus) & Oct. $21^{\text {st }} 2002$ & Kedougou (Senegal) & 5 \\
\hline HD 78788 & Human blood & Feb. $14^{\text {th }} 1991$ & Dakar (Senegal) & Unknown \\
\hline Ref. (MR 766) & Monkey & Apr. $18^{\text {th }} 1947$ & Zika Forest (Uganda) & 20 \\
\hline
\end{tabular}

Passages were conducted with Aedes (Stegomyia) pseudoscutellaris 61 cells (AP-61)

described by Rutledge et al. [22]. The infectious meal consisted of two-thirds washed rabbit erythrocytes mixed with sucrose and fetal bovine serum (FBS) and one-third viral suspension. Adenosine 5'-triphosphate (ATP) was added at a final concentration of $5 \times 10^{-3} \mathrm{M}$ as phagostimulant. For each infection experiment, a sample of the virus-blood suspension was taken at the end of the mosquitoes feeding and stored at $-80{ }^{\circ} \mathrm{C}$ for titration as previously described [20]. After $30 \mathrm{~min}$ of exposure, Mosquitoes were cold anaesthetized and sorted according to their stomach repletion. Fully engorged specimens were preserved and maintained at $27{ }^{\circ} \mathrm{C}$, $80 \% \mathrm{RH}, 16: 8 \mathrm{~h}(\mathrm{~L}: \mathrm{D})$ photoperiod and fed on $10 \%$ sucrose for extrinsic incubation of the virus.

At 5, 10, and $15 \mathrm{dpi}$, samples of mosquitoes were collected randomly, cold anesthesized, and their legs and wings removed and transferred individually into separate tubes. The proboscis of each mosquito was then inserted into a capillary tube containing $1 \mu \mathrm{L}$ of FBS for salivation for up to $30 \mathrm{~min}$. After salivation, each mosquito body (whole body except legs and wings) and saliva sample was placed in a separate tube. Aedes luteocephalus was tested only at 15 dpi because this species is difficult to rear, feed and maintain in the laboratory, so a small sample was available.

\section{Virus detection}

Mosquito bodies, and legs/wings were triturated using cold pestles in $500 \mu \mathrm{l}$ of L-15 medium (GibcoBRL, Grand Island, NY, USA). After trituration, pools were centrifuged at $7500 \mathrm{rpm}$ for $10 \mathrm{~min}$ at $4{ }^{\circ} \mathrm{C}$. For each sample, $100 \mu \mathrm{l}$ of supernatant were used for RNA extraction with the QiaAmp Viral RNA Extraction Kit (Qiagen, Heiden, Germany) according to the manufacturer's protocol with slight modification [23]. The RNA was amplified using a real-time RT-PCR assay and an ABI Prism 7500 SDS Real-Time apparatus (Applied Biosystems, Foster City, CA) using the QuantiTect kit (Qiagen, Hilden, Germany). The $25 \mu \mathrm{l}$ reaction volume contained $5 \mu \mathrm{l}$ of extracted RNA, $10 \mu \mathrm{l}$ of buffer (2x QuantiTect Probe), $6.8 \mu \mathrm{l}$ of RNase free water, $1.25 \mu \mathrm{l}$ of each primer, $0.5 \mu \mathrm{l}$ of probe, and $0.2 \mu \mathrm{l}$ of enzymes. The primers and probe sequences were described by Faye et al. [23].
Only RT-PCR was used to detect ZIKV because the objective of this study is only to show the competence of the vector. Thus, if we are able to show that the virus reached the saliva, it implies that the vector is competent. In our previous experiences with other viruses (West Nile, Usutu), we have noticed that RT-PCR and infectious viral particles are generally very consistent and concordant in their conclusions and trends [24, 25]. Such a trend has also been confirmed on C6/36 cells for Chikungunya virus by Chen et al. [26].

\section{Data analysis}

Infection (number of positive bodies/total number of mosquitoes tested), dissemination (number of infected legs \& wings/total number of infected bodies), and transmission (number of positive saliva/number of infected legs \& wings) rates were calculated for each species on each dpi. Rates were compared using Fisher's exact test. The associations between viral titers and infection rates were assessed by Spearman's rank-order correlation test. Differences were considered statistically significant at $p<0.05$. Statistical tests were performed using R v. 3.0.1 (R Foundation for Statistical Computing, Vienna, Austria).

\section{Results}

After exposure to virus titers ranging from $2.7 \times 10^{6}$ to $4 \times 10^{7} \mathrm{PFU} / \mathrm{ml}$ (Additional file 1: Table S1), overall, 111 (50.2 \%) of the 221 Ae. aegypti from Dakar, 216 (57.6 \%) of the 375 Ae. aegypti from Kedougou, 56 (18.7\%) of the 300 Ae. unilineatus, 37 (14.4\%) of the 256 Ae. vittatus and $45(75.0 \%)$ of the 60 Ae. luteocephalus tested were infected by the six ZIKV (Fig. 1). No correlation was found between viral titer and mosquito infection rates at 5, 10 and $15 \mathrm{dpi}$ (spearman test; rho $>-0.18, p>0.95$ ). Infection rates varied significantly between strain for each species tested at 5,10 and $15 \mathrm{dpi}(p<0.05)$. Highest infection rates were generally observed at $10 \mathrm{dpi}$ for Ae. aegypti from Dakar (3/6 viral strains), Ae. aegypti from Kedougou (4/6 viral strains) and Ae. vittatus (3/6 viral strains) and 15 dpi for Ae. unilineatus (4/6 viral strains). Aedes aegypti, Ae. unilineatus, and Ae. vittatus populations showed a decreasing trend in infection rates either from 5 to $10 \mathrm{dpi}$ or 


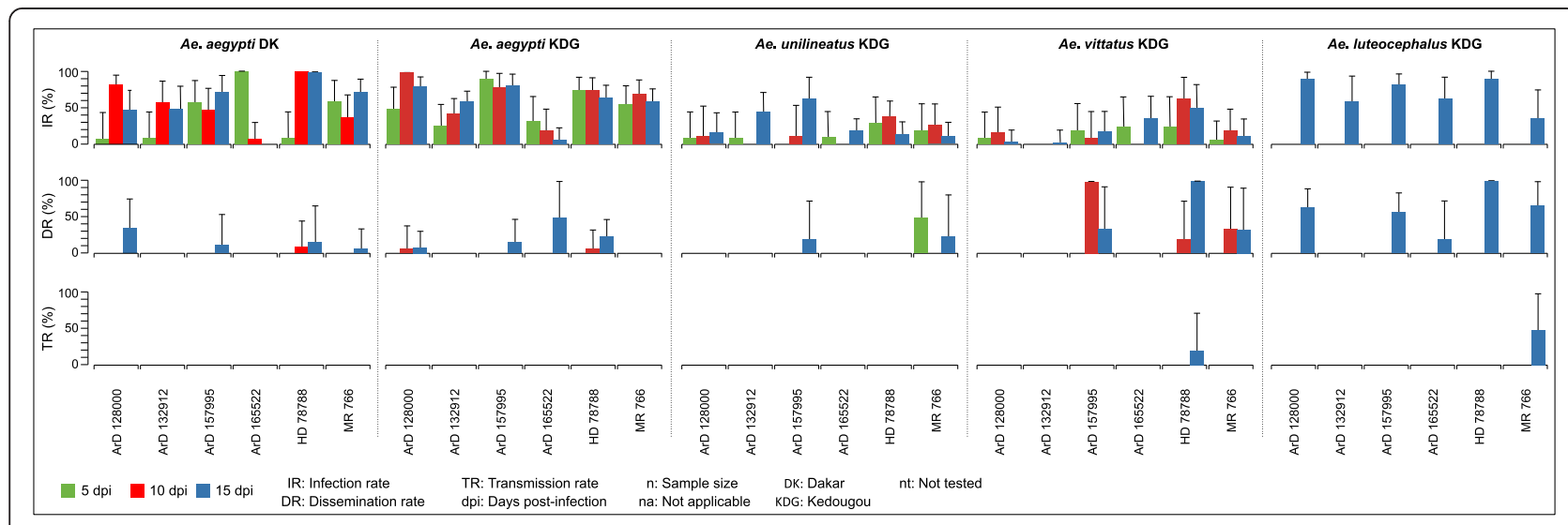

Fig. 1 Infection, dissemination and transmission rates at different incubation period for different mosquito species orally exposed with six zika virus strains

from 10 to $15 \mathrm{dpi}$ in 19 of the 24 infection assays performed.

The overall trend has been the low dissemination in the populations of Ae. aegypti from Dakar $(6.3 \%$ of the 111 specimen tested), Ae. aegypti from Kedougou (5.6\% of the 216 specimen tested) and Ae. unilineatus (5.3\% of the 56 specimen tested) and a relatively high dissemination rates in Ae. vittatus ( $27.0 \%$ of the 37 specimen tested) and Ae. luteocephalus (42.2\% of the 45 specimen tested). Only Ae. unilineatus disseminated one viral strain at $5 \mathrm{dpi}$. Dissemination rates varied between 0 and $10 \%$ for the populations of Ae. aegypti and beteween 0 and $100 \%$ for Ae. vittatus at $10 \mathrm{dpi}$. At $15 \mathrm{dpi}$, these dissemination rates varied between 0 and $50 \%$ for the populations of Ae. aegypti and beteween 0 and $100 \%$ for $A e$. vittatus and Ae. luteocephalus. Dissemination rates were statistically comparable for all species at 10 and $15 \mathrm{dpi}$ $(p>0.09)$ except Ae. luteocephalus at 15 dpi $(p=0.03)$. The 2 populations of Ae. aegypti and Ae. luteocephalus disseminated 4 of the 6 ZIKV strains tested, Ae. vittatus 3 strains and Ae. unilineatus only 2 strains.

Taken into account all the six viral strains, a total of 7 , 12, 3, 10 and 27 saliva of Ae. aegypti from Dakar, Ae. aegypti from Kedougou, Ae. unilineatus, Ae. vittatus and $A$ e. luteocephalus, respectivly were tested for the presence of ZIKV RNA. Only Ae. vittatus and Ae. luteocephalus transmitted ZIKV strains HD 78788 (20\%) and MR 766 (50\%) respectively at 15 dpi indicated by the presence of the viral genome in the mosquito saliva. These transmission rates were comparable $(p=1)$.

\section{Discussion}

Only RT-PCR was used to detect ZIKV because the objective of this study is only to show the competence of the vector. Thus, if we are able to show that the virus reached the saliva, it implies that the vector is competent. Mean $\mathrm{Ct}$ values decreased gradually from 5 to $15 \mathrm{dpi}$ for most infected species and ZIKV strain association suggesting that the mean amount of virus in each mosquito has increased and thus the RT-PCR was able to detect the ZIKV amplification (Additional file 1: Table S1).

All mosquito species tested were susceptible to ZIKV infection with infection rates varying according to viral strains and extrinsic incubation periods. In some cases, these rates were as high as those obtained with Ae. aegypti and Ae. albopictus populations from Singapore [27].

Our results also revealed globally in Ae. aegypti, Ae. unilineatus, and Ae. vittatus populations a decreasing trend in infection rates either from 5 to $10 \mathrm{dpi}$ or from 10 to $15 \mathrm{dpi}$. Indeed, in 24 infection assays performed, 17 showed this profile. Similar profile were also observed in Ae aegypti populations from Singapore between 6 and $7 \mathrm{dpi}$ and those of Ae. albopictus from 6 to $10 \mathrm{dpi}$ $[27,28]$. This decrease following high infection rates obtained at 5 or 10 dpi suggest that ZIKV infection could induce an immune response that can lead to replication of the virus at undetectable levels.

The main findings of our study was the low dissemination and lack of transmission of the population of Ae. aegypti tested here. This result was unexpected taken into account very high transmission rates obtained in previous studies with this species in Senegal and Asia. Indeed, a previous study had shown that $A e$. aegypti from Kebemer (a locality near Dakar, Senegal), were competent to ZIKV with a transmission rate of $88 \%$ at 7 dpi [15], reaching as high as $95 \%$ at $21 \mathrm{dpi}$. However, intrathoracic inoculation and transmission to newborn mice used in that study were different from our methods. With other viruses, intrathoracic inoculation is well known to bypass the midgut infection barrier, leading to a shorter period of extrinsic incubation, a direct exposure of the virus to the salivary glands, and thereafter generally more efficient infection and transmission [29]. 
The recent studies conducted on Ae. aegypti populations from Singapore using an oral infection methods similar to our method have generated high salivary glands infection rates of 62 and $100 \%$ at 5 and $10 \mathrm{dpi}$, respectivly [27].

The low or lack of transmission we observed could not be attributed to the transmission method used (salivation on capillary tubes) which efficacy has been proven. Indeed, this system has been recently used to demonstrate vector competence of Ae. albopictus for the same ZIKV [28]; but also with various other mosquito species tested for various viruses [21, 30]. In addition, a comparative study has shown that Ae. albopictus and Ae. taeniorhynchus transmit better with the method of capillary tubes than with the use of an animal for transmission monitoring [31]. Because the experiments were carried out at $27{ }^{\circ} \mathrm{C}$, the average temperature in Senegal during ZIKV transmission, the temperature may not be impacting our transmission results. Also the titers of the ZIKV we used could not be responsible because a virus suspension at a final concentration of $7.0 \log _{10}$ tissue culture infectious dose $\left(\mathrm{TCID}_{50}\right) / \mathrm{ml}$ were sucessfully used in previous mosquito experiments [27, 28]. Our low transmission results were also not due to the detection method, since the real-time RT-PCR has often greater sensitivity than the other methods [32].

The difference between the transmission rates observed in this study and the others could be explained by genetic variability between Ae. aegypti populations from different geographical origin. The impact of this variability in vector competence results was demonstrated for several vector/ virus associations [33, 34]. Although the lack of transmission by Ae. aegypti populations was not expected, this is consistant with the low number of ZIKV strains isolated from this species in West Africa. Indeed only 2 strains of ZIKV were isolated from this species in Senegal, one strain in Burkina Faso and one in Ivory Coast $[35,36]$.

The low competence of Ae. vittatus and Ae. luteocephalus is discordant with the high abundance of these species and their frequent association with ZIKV in the field $[19,35,36]$. Indeed, Ae. vittatus, with $22.98 \%$ of the fauna, was the most abundant mosquito species collected by human landing catch in the Kedougou area between June 2009 and December 2010. This species has beeen found associated with ZIKV in Ivory Cost ( 2 strains) and Senegal (15 isolates at least) in West Africa. Ae. luteocephalus is generally less abundant than Ae. vittatus but more ZIKV was isolated in this species in Burkina Faso (40 strains), Ivory Cost (48 strains) and Senegal (92 strains).

However, an Ae. aegypti population with poor vector competence but high density have been shown to be the principal vector of a Yellow Fever outbreak in Nigeria [37].
The low infection and dissemination rates and the lack of transmission by Ae. unilineatus are in agreement with the single isolation of ZIKV from this species in nature and its low representation from human landing catch collection [38].

Our results are discordant with the pattern of ZIKV transmission in Southeastern Senegal. Indeed ZIKV has the highest frequency of detections among arboviruses found in this area. Its amplifications have been detected during 20 of the 34 years of monitoring that took place between 1972 and 2005 there. Furthermore, the virus has been isolated continuously every year from 1984 to 1994 [35]. Our low transmission rate are difficult to reconcile with continuous ZIKV transmission, and suggest the involvement of other vectors or other mechanisms of maintenance and transmission. The involvment of other vectors is supported by the fact that lesser number of strains were isolated from the tested species compared to Ae. furcifer and Ae. taylori [35, 36]. Vector competence of these species should be investigated to better understand ZIKV epidemiology and transmission in Senegal. Our results suggest that the species tested here are probably less implicated in the regular ZIKV amplifications in Senegal. Other mechanisms of maintenance and transmission probably include the vertical and/or venereal transmission of the virus supported by its detection in several pools of male Ae. furcifer in nature $[35,36]$. This phenomenon, already demonstrated in nature has never been proven experimentally and therefore needs further investigation.

\section{Conclusion}

All the populations of Ae aegypti, Ae. unilineatus, Ae. vittatus, and Ae. luteocephalus tested here were susceptible to oral infection of ZIKV but only a low proportion of Ae. vittatus and Ae. luteocephalus had the viral genome in their saliva and thus the potential to transmit the virus. Vector competence and vertical transmission studies involving other species more often associated with ZIKV should be undertaken to better understand the ecology and epidemiology of this arbovirus of growing medical interest.

\section{Additional file}

Additional file 1: Table S1. Ct (threshold cycle) mean values for each mosquito species body (the whole body except legs and wings) and Zika virus strain at different days post-infection (dpi). (DOC 73 kb)

\section{Abbreviations}

Ae.: Aedes; ATP: Adenosine 5'-triphosphate; dpi: days post-infection; FBS: Fetal bovine serum; IgM: Immunoglobuline M; NHPs: Non-human primates; PFU: Plaque forming unit; RH: Relative humidity; RNA: Ribonucleic Acid; RT-PCR: Reverse transcription-polymerase chain reaction; $\mathrm{TCID}_{50}$ : $50 \%$ tissue culture infectious dose; ZIKV: Zika virus. 


\section{Competing interests}

The authors declare that they have no competing interests.

\section{Authors' contributions}

CTD, DD, AAS, SCW and MD conceived and designed the study. CTD, AG and DD collected the mosquito populations. CTD, AG, OumF, AAS and OusF performed the laboratory experiments. $C T D, D D$, and MD analyzed the data and drafted the manuscript. CTD, DD, YB, ID, OumF, AAS, OusF and MD critically revised the manuscript. All authors read and approved the final manuscript.

\section{Acknowledgements}

This work was supported by grant from the US National Institute of Allergy and Infectious Diseases [Grant R01Al069145] of the National Institutes of Health. The authors gratefully thank Amadou Thiaw, Abdou K. Bodian for their technical assistance during fieldwork and mosquito rearing.

\section{Author details BP 220, Dakar, Senegal. Unité des Arbovirus et Virus de Fièvres Senegal. ${ }^{3}$ Département de Biologie Animale, Laboratoire d'Écologie USA. \\ Received: 24 April 2015 Accepted: 19 October 2015 Published online: 02 November 2015}

'Unité d'Entomologie Médicale, Institut Pasteur de Dakar, 36 Avenue Pasteur, Hémorragiques, Institut Pasteur de Dakar, 36 Avenue Pasteur, BP 220, Dakar, Vectorielle et Parasitaire, Université Cheikh Anta Diop, Dakar, Senegal. ${ }^{4}$ Institute for Human Infections and Immunity, Center for Biodefense and Emerging Infectious Diseases, University of Texas Medical Branch, Galveston, TX 77555-0610, USA. ${ }^{5}$ Department of Pathology and Microbiology \& Immunology, University of Texas Medical Branch, Galveston, TX 77555-0610,

\section{References}

1. Dick GW, Kitchen SF, Haddow AJ. Zika virus. I. Isolations and serological specificity. Trans R Soc Trop Med Hyg. 1952;46(5):509-20.

2. Wolfe ND, Kilbourn AM, Karesh WB, Rahman HA, Bosi EJ, Cropp BC, et al. Sylvatic transmission of arboviruses among Bornean orangutans. Am J Trop Med Hyg. 2001;64(5-6):310-6.

3. Simpson DI. Zika Virus Infection in Man. Trans R Soc Trop Med Hyg. 1964:58:335-8.

4. Karabatsos N. International catalogue of arboviruses. 3rd ed. San Antonio: American Society of Tropical Medicine and Hygiene; 1985.

5. Duffy MR, Chen T-H, Hancock WT, Powers AM, Kool JL, Lanciotti RS, et al. Zika virus outbreak on Yap Island, federated states of Micronesia. N Engl J Med. 2009;360(24):2536-43.

6. Hayes EB. Zika virus outside Africa. Emerg Infect Dis. 2009;15(9):1347-50. doi:10.3201/eid1509.090442.

7. Olson JG, Ksiazek TG, Suhandiman, Triwibowo. Zika virus, a cause of fever in Central Java, Indonesia. Trans R Soc Trop Med Hyg. 1981;75(3):389-93.

8. Darwish MA, Hoogstraal H, Roberts TJ, Ghazi R, Amer T. A seroepidemiological survey for Bunyaviridae and certain other arboviruses in Pakistan. Trans R Soc Trop Med Hyg. 1983;77(4):446-50.

9. Monlun E, Zeller H, Le Guenno B, Traoré-Lamizana M, Hervy JP, Adam F, et al. Surveillance of the circulation of arbovirus of medical interest in the region of eastern Senegal. Bull Soc Pathol Exot. 1993;86(1):21-8.

10. Cao-Lormeau V-M, Musso D. Emerging arboviruses in the Pacific. Lancet. 2014;384(9954):1571-2.

11. Musso D, Nilles EJ, Cao Lormeau VM. Rapid spread of emerging Zika virus in the Pacific area. Clin Microbiol Infect. 2014;20(10):0595-6.

12. Fagbami AH. Zika virus infections in Nigeria: virological and seroepidemiological investigations in Oyo State. J Hyg (Lond). 1979;83(2):213-9.

13. Marchette NJ, Garcia R, Rudnick A. Isolation of Zika virus from Aedes aegypti mosquitoes in Malaysia. Am J Trop Med Hyg. 1969;18(3):411-5.

14. Boorman JP, Porterfield JS. A simple technique for infection of mosquitoes with viruses; transmission of Zika virus. Trans R Soc Trop Med Hyg. 1956;50(3):238-42.

15. Cornet M, Robin Y, Adam C, Valade M, Calvo MA. Transmission expérimentale comparée du virus amaril et du virus Zika chez Aedes aegypti. Cah ORSTOM ser Ent med et Parasitol. 1979;17:47-53.
16. Berge TE. International Catalog of Arboviruses. 2nd ed. Washington, D.C: National Institute of Allergy and Infectious Diseases and the Center for Disease Control; 1975.

17. McCrae AW, Kirya BG. Yellow fever and Zika virus epizootics and enzootics in Uganda. Trans R Soc Trop Med Hyg. 1982;76(4):552-62.

18. Akoua-Koffi C, Diarrassouba S, Benie VB, Ngbichi JM, Bozoua T, Bosson A, et al. Investigation surrounding a fatal case of yellow fever in Cote d'Ivoire in 1999. Bull Soc Pathol Exot. 2001;94(3):227-30.

19. Diallo D, Sall AA, Buenemann M, Chen R, Faye O, Diagne CT, et al. Landscape ecology of sylvatic chikungunya virus and mosquito vectors in southeastern Senegal. PLoS Negl Trop Dis. 2012;6(6):e1649. doi:10.1371/journal.pntd.0001649.

20. De Madrid AT, Porterfield JS. A simple micro-culture method for the study of group B arboviruses. Bull World Health Organ. 1969;40(1):113-21.

21. Diagne $C T$, Faye $O$, Guerbois $M$, Knight $R$, Diallo D, Faye $O$ et al. Vector Competence of Aedes aegypti and Aedes vittatus (Diptera: Culicidae) from Senegal and Cape Verde Archipelago for West African Lineages of Chikungunya Virus. Am J Trop Med Hyg. 2014. doi:10.4269/ajtmh.13-0627.

22. Rutledge LC, Ward RA, Gould OJ. Studies on the feeding response of mosquitoes to nutritive solutions in a new membrane feeder. Mosq News. 1964;24:407-19.

23. Faye O, Faye O, Diallo D, Diallo M, Weidmann M, Sall AA. Quantitative realtime PCR detection of Zika virus and evaluation with field-caught mosquitoes. Virol J. 2013;10:311. doi:10.1186/1743-422X-10-311.

24. Nikolay B, Diallo M, Faye O, Boye CS, Sall AA. Vector competence of Culex neavei (Diptera: Culicidae) for Usutu virus. Am J Trop Med Hyg. 2012;86(6):993-6.

25. Fall G, Diallo M, Loucoubar C, Faye O. Vector competence of Culex neavei and Culex quinquefasciatus (Diptera: Culicidae) from Senegal for lineages 1 , 2, Koutango and a putative new lineage of West Nile virus. Am J Trop Med Hyg. 2014;90(4):747-54

26. Chen R, Wang E, Tsetsarkin KA, Weaver SC. Chikungunya virus $3^{\prime}$ untranslated region: adaptation to mosquitoes and a population bottleneck as major evolutionary forces. PLoS Pathog. 2013;9(8):e1003591.

27. Li MI, Wong PS, Ng LC, Tan CH. Oral susceptibility of Singapore Aedes (Stegomyia) aegypti (Linnaeus) to Zika virus. PLoS Negl Trop Dis. 2012;6(8):e1792. doi:10.1371/journal.pntd.0001792.

28. Wong PS, Li MZ, Chong CS, Ng LC, Tan CH. Aedes (Stegomyia) albopictus (Skuse): a potential vector of Zika virus in Singapore. PLoS Negl Trop Dis. 2013;7(8):e2348. doi:10.1371/journal.pntd.0002348.

29. Smith DR, Carrara A-S, Aguilar PV, Weaver SC. Evaluation of methods to assess transmission potential of Venezuelan equine encephalitis virus by mosquitoes and estimation of mosquito saliva titers. Am J Trop Med Hyg. 2005;73(1):33-9.

30. Anderson SL, Richards SL, Smartt CT. A simple method for determining arbovirus transmission in mosquitoes. J Am Mosq Control Assoc. 2010;26(1):108-11.

31. Smith DR, Aguilar PV, Coffey LL, Gromowski GD, Wang E, Weaver SC Venezuelan equine encephalitis virus transmission and effect on pathogenesis. Emerg Infect Dis. 2006;12(8):1190-6. doi:10.3201/eid1208.050841.

32. Faye $\mathrm{O}, \mathrm{Ba} Y$, Faye O, Talla C, Diallo D, Chen R, et al. Urban epidemic of dengue virus serotype 3 infection, Senegal, 2009. Emerg Infect Dis. 2014;20(3):456-9. doi:10.3201/eid2003.121885.

33. Tesh RB, Gubler DJ, Rosen L. Variation among geographic strains of Aedes albopictus in susceptibility to infection with chikungunya virus. Am J Trop Med Hyg. 1976;25(2):326-35.

34. Gubler DJ, Nalim S, Tan R, Saipan H, Sulianti SJ. Variation in susceptibility to oral infection with dengue viruses among geographic strains of Aedes aegypti. Am J Trop Med Hyg. 1979;28(6):1045-52.

35. Adam F, Digoutte JP. Virus d'Afrique (Base de Données). Centre Collaborateur OMS de Référence et de Recherche pour les Arbovirus et les Virus de Fièvres Hémorrhagiques. In: CRORA. 2013. Available at http://www.pasteur.fr/ recherche/banques/CRORA/. Accessed April 2, 2013.

36. Diallo D, Sall AA, Diagne CT, Faye O, Faye O, Ba Y, et al. Zika Virus Emergence in Mosquitoes in Southeastern Senegal, 2011. PLoS One. 2014;9(10):e109442.

37. Miller BR, Monath TP, Tabachnick WJ, Ezike VI. Epidemic yellow fever caused by an incompetent mosquito vector. Trop Med Parasitol. 1989;40(4):396-9.

38. Diallo D, Sall AA, Diagne CT, Faye O, Hanley KA, Buenemann M, et al. Patterns of a sylvatic yellow fever virus amplification in southeastern Senegal, 2010. Am J Trop Med Hyg. 2014;90(6):1003-13. 